\title{
MEMORAR LA CULTURA: MODOS DE MANTENER Y FORMAR LAS IDENTIDADES MAYAS MODERNAS
}

\author{
Felix A. KupPrat \\ Universität Bonn
}

\begin{abstract}
RI TZIIJ RE LE'A'J: Memoria (e jun k'ayewaal tziij wa'lib'eem loq) e jun tziij k'o chi kik'u'x ri tikaweek jay e jun ch'a'teem kuk'am uwach re ri utziil chomaliil pa ri tinamit Iximuleew pa lo ri wo'lajuuj junaab'. E chwi ri tziij k'o chi kik'u'x ri motza'j tikaweex, e kunuk' wii riib' ri katz'iyaqiil ri motza'loq tikaweex. Jay lik e kuchap wii riib' che ri uyakiik jay ri uch'apab'exiik che taq ri ano'n.

Wa jun le'aj kintz'iibaj kuchap riib' ruuk' ri tziij pa nima ronojee motza’j tikaweex jay xa pa ri tinamit. Kak'amb'ex ub'ee ruuk' ri na'ooj chwi ri chaak kaki'an ri tikaweex maya' jay ri ukuxtaxiik ri k'axk'oliil xkiwa' lib'eej ri tikaweex maya' xa pa ri tinamit. E ruma ri' lik chi rajawaxiik kaq'alajisax ri ano'n re wa'ora ruuk' ri ano'n re ojeer loq ke ri tikaweex maya'. Ri ano'n re wa'ora kuchap riib' ruuk' ri ano'n xa re, pa ri tinamit jay ri ano'n re ojeer loq, lik chi rajawaxiik kayak uwach pacha' kuya uchuq'aab' ri ano'n re wa'ora. Pa k'isb'al re, ri motza’j xa re pa taq tinamit jay ri pa nima ronojee motza’j tikaweex, lik kaq'alajin uwach ruma ri kich'ob'onik jay ri uchomoxiik ri kik'aslemaal chi kiwach.
\end{abstract}

Chi Rajawaxik RI tzIIJ: Kuxtab'al tziij, Maya'iib‘ taq chi tinamit, Jumajwach, Panima', Iximuleew.*

Resumen: Memoria ha sido una palabra clave en el proceso de paz en Guatemala durante los últimos quince años. De acuerdo con teorías de la memoria colectiva, ésta forma la identidad de grupos y es sustancial para la construcción y el mantenimiento de la cultura. Al contrastar el movimiento maya con estrategias locales para la conmemoración de las violaciones de los derechos humanos durante el conflicto armado, este artículo conecta el concepto de etnicidad a nivel interregional con la identidad étnica local. Por lo tanto, se propone una división entre los marcadores étnicos sincrónicos y los asincrónicos de la etnicidad maya: el primero se asocia con elementos culturales locales y el segundo con procesos de revitalización. Finalmente, se plantea que los rasgos estructurales, como la memoria cultural, deben ser considerados marcadores étnicos en ambos niveles: el local y el interregional.

Palabras clave: Memoria colectiva, etnicidad maya, identidad cultural, Río Negro, Guatemala.

AвSTRACT: Memory has been a keyword for the peace process in Guatemala in the last fifteen years. According to theories on collective memory it shapes group identities and is crucial for the construction and maintenance of culture. Contrasting maya cultural activism and local commemorative strategies dealing with human rights violations during the civil war, this paper aims to connect ethnicity on an inter-regional and ethnic identity on a local level. Therefore a division will be made between synchronous and asynchronous ethnic markers of pan-maya identity, associating the former with cultural elements on a local level and the latter with revitalization processes. Finally it is proposed that structural features such as cultural memory should be considered crucial ethnic markers on both levels: the local and the inter-regional.

KEYwords: Collective Memory, Maya Ethnicity, Cultural Identity, Rio Negro, Guatemala.

RECEPCIÓN: 17 de septiembre del 2010.

ACEPTACIÓN: 15 de marzo del 2011.

* Traducido por Máximo Chen Siana y Elsira Cahuec Valey; véase la nota de agradecimiento. 



\title{
MEMORAR LA CULTURA: MODOS DE MANTENER Y FORMAR LAS IDENTIDADES MAYAS MODERNAS"
}

\author{
Felix A. Kupprat \\ Universität Bonn
}

\section{Introducción}

En los contextos de las sociedades pluriétnicas, los conflictos étnicos y los estados multinacionales, el concepto de la etnicidad ha sido objeto de varios acercamientos multi- e interdisciplinarios dirigidos hacia una explicación de este fenómeno social, cultural y político.

En el mundo maya actual, tanto en Chiapas como en la península de Yucatán (México), en Belice y en Guatemala, la identidad maya y la etnicidad como concepto afín son pilares de movimientos sociopolíticos y culturales - lo que también se ha discutido en varias investigaciones etnográficas y antropológicas (cf. Fischer y Brown, 1996; Nelson, 1996; Warren, 1998; Bastos y Camus, 2003; Macleod, 2006; Sachse, 2006; Bastos y Cumes, 2007; Alejos García, 2008: 199205) - . Las palabras claves como maya, identidad o cultura forman la base de argumentos poderosos, pero pocas veces los mismos discursos ofrecen un modelo operacional para el manejo de estos términos.

En este artículo se discutirá la identidad maya como se define en el Acuerdo sobre identidad y derechos de los pueblos indígenas (Naciones Unidas y Unidad Revolucionaria Nacional Guatemalteca, 1995) y cómo es aplicada por los activistas del movimiento maya en Guatemala. En lo que sigue, se contrapon-

\footnotetext{
Debo en primer lugar dar gracias a toda la comunidad de Río Negro que me recibe con gran hospitalidad y me apoya tanto personalmente como con respecto a mis investigaciones: igracias por compartir! En especial quiero mencionar a la familia de Sebastián Iboy Osorio y Magdalena Alvarado, quienes comparten incondicionalmente su hogar y me hacen sentir parte de él.

Dos personas a quienes agradezco mucho su amabilidad, su paciencia y la traducción del resumen a la lengua achi son mis maestros de dicho idioma, Máximo Chen Siana y Elsira Cahuec Valey. Por su cooperación en Rabinal quiero expresar mis gracias a Carlos Chen Osorio, Guillermo Chen Morales y Eleodoro Osorio Sanchez.

Por la revisión del artículo, sus correcciones y comentarios le quiero expresar mis gracias a Verónica A. Vázquez López. Por su ayuda con la organización de la investigación y sus comentarios productivos les debo mis gracias a Kathrin Weber, José Victor Lindenmayer, Sucely Ical Lem, Lars Frühsorge, Frauke Sachse, Nikolai Grube y a todas las personas que me han apoyado en el camino de la investigación. Por sus valiosos consejos y comentarios críticos agradezco a ErikoYamasaki y José Alejos García.
} 
drá esta perspectiva macroscópica a un estudio de caso con enfoque local describiendo la identidad étnica en la aldea Río Negro, Rabinal, Baja Verapaz, que se define notablemente por la construcción de una memoria colectiva. Se mostrarán las diferencias cualitativas entre los niveles locales y globales de la identidad y se hará una propuesta para el manejo metodológico de términos y conceptos.

\section{Etnicidad e identidad étnica}

Un grupo étnico normalmente se ha definido a través de varios elementos críticos; los más comunes son una cultura compartida y la creencia en una descendencia común. Max Weber definió los grupos étnicos como:

[...] aquellos grupos humanos que, fundándose en la semejanza del hábito exterior y de las costumbres, o de ambos a la vez, o en recuerdos de colonización y migración, abrigan una creencia subjetiva en una procedencia común, de tal suerte que la creencia es importante para la ampliación de las comunidades; pero la designaremos así siempre que no representen 'clanes', aunque sin tener en cuenta si existe o no una verdadera comunidad de sangre (Weber, 1964: 318).

Este punto de vista se ha conservado en varios discursos sobre la etnicidad, aunque implica que entonces el concepto de etnia se basa en una creencia normalmente no verificable. En los años sesenta, Frederik Barth (1969: 10-15) dio un impulso importante a ese modelo constructivista, definiendo al grupo étnico como un grupo socialmente organizado y legitimado como tal a través de criterios culturales que dependen del caso particular. Últimamente se han tomado en cuenta varias opciones para combinar conceptos primordiales con el modelo de lo étnico considerado como construcción social, sobre todo mediante nuevos acercamientos hacia la pluriculturalidad (vg. Yinger, 1985: 159; para el area maya véase Fischer et al., 1999).

En este discurso teórico se nota que los términos identidad étnica y etnicidad a menudo han sido usados indiferenciadamente. Por eso propongo una distinción funcional entre estos dos conceptos que forman la base de la siguiente discusión:

La identidad étnica es la parte de la autopercepción que comparten todos los miembros de un grupo étnico. Se construye mediante la interacción intra- y, necesariamente, interétnica, porque una identidad funcionalmente delimita lo propio de lo ajeno. La distinción se logra por medio de marcadores específicos (que tipológicamente varían entre culturas diferentes), como traje, lenguaje, expresiones espirituales, organización social, etc.; estos crean un sentimiento de "nosotros" en oposición con "los otros". Dichos marcadores étnicos no son indicadores de autenticidad, pero sí están cargados de una asociación común de permanencia a un grupo particular en un contexto pluricultural. 
La etnicidad es una forma especial de la identidad étnica que busca definirse por sus propios rasgos culturales de manera positivista, así que se expresa de manera consciente y reflexiva. Esto implica que los marcadores étnicos se vuelven explícitos y normativos, formando parte de una ideología de la autoidentificación.

Un caso popular debe ilustrar cómo se normalizan marcadores étnicos, en este caso a nivel político, cruzando acercamientos constructivistas y esencialistas: el Acuerdo sobre identidad y derechos de los pueblos indígenas (Naciones Unidas / Unidad Revolucionaria Nacional Guatemalteca, 1995), firmado entre la URNG y el gobierno guatemalteco durante el proceso de las negociaciones de paz, da una definición de la identidad indígena y especialmente de la identidad maya en Guatemala. Declara: "La identidad de los pueblos es un conjunto de elementos que los definen y, a su vez, los hacen reconocerse como tal". Luego se especifican cinco marcadores para la identidad maya en particular:

I) Descendencia directa de los mayas antiguos.

II) Una lengua de raíz común.

III) Una cosmovisión común.

IV) Una cultura común.

V) La autoidentificación.

Sistemáticamente, estos puntos se pueden resumir en tres categorías: 1) descendencia, 2) cultura y 3) autoidentificación; considero a la lengua y la cosmovisión como aspectos culturales, entendiendo cultura como un sistema de símbolos compartidos que organiza una sociedad (Geertz, 1973: 5).

Primero, la descendencia directa de los antiguos mayas (1) —es decir, de una población prehispánica - es algo que prácticamente, considerando el caso de un individuo en particular, no se puede comprobar (Yinger, 1985: 162). En ese argumento se reflejan discursos esencialistas que juzgan a la identidad étnica como algo biológica o históricamente determinado, lo cual reduce el concepto de etnia, en su forma extrema, a raza o casta, respectivamente.

Segundo, la autoidentificación (3) implica la idea irreal de poseer la libertad individual de escoger una identidad por deseo personal. Por un lado, la identidad no es cuestión de voluntad. La percepción de sí mismo siempre es influida gravemente por la percepción de los otros y en la construcción de la identidad el individuo y el colectivo siempre se retroalimentan. Por eso, una persona no simplemente puede decidir ser maya, sino en primer lugar tendrá que ser reconocida como tal por la comunidad. Por otro lado, a veces los individuos y los grupos niegan una identidad propia; empero, no logran eliminarla porque siguen siendo identificados mediante ella por los demás —un fenómeno frecuentemente observado en contextos migratorios y globalizadores-.

La definición dada de identidad claramente se orienta a los debates académicos tomando en cuenta argumentos claves de discursos distintos. Así, vemos que 
el Acuerdo busca implementar ideas esencialistas (1: descendencia) tanto como constructivistas (3: auto-identificación) en su definición de la identidad maya. En fin, el presupuesto de que la identidad se define a través de la cultura (2) no puede ser considerada un punto de vista esencialista ni constructivista pues se deben aplicar consideraciones importantes de ambos discursos. Por un lado, cultura (considerada como un sistema semiótico) y todo lo que implica es asimilable por principio. Los símbolos pueden ser aprendidos, igual que sus relaciones y su aplicación. Por eso, como ya reconoció Barth (1969), las fronteras étnicas basadas en la cultura son permeables. Sin embargo, por el otro lado, la cultura está determinada por el contexto social. La cultura materna de un individuo o un grupo es aquella aprendida desde niño y que para el resto de la vida suele jugar un papel importante. Por ejemplo, Edward Fischer junto con otros investigadores (Fischer et al., 1999; Fischer, 2001), tomando una posición "anti-antiesencialista", muestra que la cultura incluye aspectos cognitivos frecuentemente inconscientes. Entonces, la identidad cultural, es decir, la cultura a la que pertenece uno, es determinada por estructuras sociales y, en casos donde la cultura se trasmite básicamente mediante la familia, también por la descendencia (aunque, bajo ciertas condiciones, las fronteras culturales pueden ser cruzadas).

Claramente, interpretando el Acuerdo como testimonio de los conceptos aplicados de identidad y etnicidad, es necesario mencionar que los fines de su redacción son de carácter político, dirigidos a lograr reformas constitucionales y legislativas. El texto refleja las actitudes y los esfuerzos del movimiento maya, promoviendo la idea de una identidad pan-maya (Bastos y Camus, 2003: 135136). Entonces, el concepto de etnicidad, descrito en el texto como identidad indígena o maya, debe ser entendido más bien en un sentido normativo. Aquí, la etnicidad no es un modelo para describir diferencias sociales (éticas), sino un conjunto de valores culturales (émicos). Para el movimiento maya eso legitima su demanda de solidaridad y organización entre todas las comunidades clasificadas como indígenas, especialmente las mayas.

\section{Marcadores étnicos sincrónicos y asincrónicos}

En el caso del movimiento maya, cuyo pensamiento se refleja fuertemente en el contenido del Acuerdo, los marcadores culturales provienen de estructuras sincrónicas y asincrónicas. Los marcadores sincrónicos suelen integrarse a la etnicidad a través de las culturas actuales, mientras los marcadores asincrónicos constituyen reconstrucciones históricas provenientes de culturas temporales y, a veces, espacialmente ajenas. Por ejemplo, el traje tradicional es un marcador sincrónico. El corte o el huipil sirven a los activistas mayas para acentuar su etnicidad (Otzoy, 1996: 154). Sin embargo, este indicador se origina en las comunidades contemporáneas, donde no sólo distingue asociativamente a los indígenas de los ladinos: a través de diseños y estilos, también distingue a grupos 
indígenas entre sí, aunque hoy día frecuentemente se mezclan los estilos locales (Otzoy, 1996: 153). Por otro lado, el uso de la escritura jeroglífica hoy día es buen ejemplo para un marcador asincrónico. Este sistema, que se vuelve más y más popular, se basa en aquella parte de la escritura de los periodos Clásico y Posclásico (y en menor medida del Preclásico) que se ha logrado descifrar en décadas muy recientes, después de siglos en desuso (Sturm, 1996: 117-119). En la aplicación actual de esta escritura se usan logogramas (incluyendo el sistema numérico) y silabogramas, también modificados y estandarizados para facilitar la representación de fonemas de las lenguas mayas de las Tierras Altas que no existen en el registro epigráfico (Sturm, 1996: 120-121). No obstante, el recurso de la escritura no proviene de las culturas locales actuales, sino de investigaciones académicas externas (Schele y Grube, 1996; Sturm, 1996: 128). Sin embargo, se entiende como elemento original y valorado de la propia cultura, implicando que su desaparición se debe a los mecanismos colonizadores represivos.

\section{La memoria como marcador étnico}

Un marcador importante de la etnicidad maya, que también se menciona en el Acuerdo, es la memoria, ya que tanto el individuo como el grupo solamente son capaces de definirse si tienen una historia, un origen y un desarrollo; una visión del pasado que los ubica en el presente. En la actualidad se pueden observar cambios fuertes en las identidades culturales de Guatemala a través de este marcador, debido a las difíciles experiencias colectivas de la población indígena durante el conflicto armado interno, las cuales hoy juegan un papel importante en las dinámicas sociales.

La memoria (tanto a nivel individual como colectivo) es un conjunto de recuerdos. Estos últimos deben entenderse como resultado de los procesos del recordar. Hay que estar conscientes de que recordar no sólo implica acceder a datos almacenados en un medio como el cerebro humano, sino es un proceso social. Los recuerdos se producen y reproducen funcionalmente en situaciones específicas de la interacción humana, combinando la información disponible del pasado con los objetivos sociales actuales (Welzer, 2002: 20). Los recuerdos que se repiten de forma similar interactúan con otros recuerdos y forman un conjunto de recuerdos relacionados: una memoria.

Distinguir entre memoria individual y colectiva a menudo resulta difícil. Por un lado, la memoria colectiva se puede entender como resultado de la fusión de los recuerdos de varios individuos. Por el otro lado, los recuerdos de un individuo frecuentemente son generados en interacción directa o indirecta con otro(s) individuo(s). Así, las memorias individuales son sumamente influidas por la memoria colectiva; ésta frecuentemente provee un contexto, un enfoque o un modelo que determinan los recuerdos personales (Halbwachs, 1992: 38; Olick, 1999: 341). 
Jan Assmann (1992: 50-52) ha distinguido dos formas de la memoria colectiva: la comunicativa y la cultural. La memoria comunicativa debe ser entendida como una memoria generacional que refiere la historia reciente y la realidad social. Ésta es completada por la memoria cultural, la cual contiene aquellas tradiciones en las que se basa el sistema semiótico conformador de una cultura. Por esta propiedad, el concepto de Assmann es altamente funcional para un entendimiento de la etnicidad como identidad cultural. Mientras la memoria comunicativa es de alta importancia para una o unas pocas generaciones, la memoria cultural se caracteriza por un proceso de fina selección y contenidos más estables. La memoria comunicativa contiene recuerdos de la cultura reciente: eventos, personajes, chismes, anécdotas; pero también, por ejemplo, cualquier elemento de la cultura popular, así que solamente pocos de sus contenidos logran una conservación temporalmente más estable. La memoria cultural se define por la formación de un canon de elementos culturales, aquellos considerados importantes para la sociedad y, por eso, trasmitidos durante muchas generaciones y frecuentemente mantenidos por instituciones de formación especializada o de educación. Mientras en las culturas orales la memoria comunicativa y la memoria cultural están divididas temporalmente por un "vacío flotante" — como ha mostrado Jan Vansina (1985) acerca de las tradiciones orales africanas-, hay que considerar que en culturas literarias y multimedias que crean acervos la división es más bien gradual. Elementos de la memoria comunicativa entran al canon de la memoria cultural, así que el canon en sí está en un estado de cambio continuo.

Al mismo tiempo, como ha constatado Aleida Assmann, el proceso de recordar siempre está conectado con el olvido (Assmann, 2008: 97-99). De hecho, olvidar es normal e inevitable, mientras recordar es la excepción. Sin embargo, uno puede olvidar de dos maneras distintas: pasiva- o activamente. El olvido pasivo se refiere al olvido no intencional, al proceso de filtración de información en que diariamente la mayoría de la información que procesamos es clasificada como irrelevante y nunca forma parte de una memoria propia. Opuesto a este mecanismo está el olvido activo que destruye, oprime y niega recuerdos. En este caso, los recuerdos son de importancia, pero actores externos o los intereses del mismo individuo o grupo buscan eliminarlos.

Justamente en este sentido la "memoria" juega un papel importante en el discurso de la historia guatemalteca reciente. Investigaciones publicadas sobre las violaciones de los derechos humanos durante el conflicto armado han ocupado el término como sinónimo de una historiografía basada en testimonios de testigos contemporáneos, lo que se puede interpretar como un esfuerzo para enfrentar el olvido pasivo y activo de la violencia. El título de la Comisión para el Esclarecimiento Histórico — "Memoria del Silencio" (сен, 1999)— y la formación del Proyecto Interdiocesano de "Recuperación de la Memoria Histórica" (Remh, 1998) son solamente dos ejemplos. En este contexto memoria se convierte en algo más que en un simple sinónimo de un método histórico: se vuelve un objeto hasta este momento política y socialmente oprimido cuya documentación, publi- 
cación o "recuperación" se establece como ideal político para los movimientos sociales; su fin es establecer justicia y prevenir la repetición de la historia. Esto se refleja bien en la oración temática popular "nunca más" — a veces ya usada en su forma sustantivizada "el nunca más"-, la cual es sinónimo del proceso de esclarecimiento a través de la documentación de las violaciones de los derechos humanos en varios países latinoamericanos.

\section{El caso de Río Negro, Guatemala ${ }^{1}$}

Para ilustrar el funcionamiento de la memoria como marcador de la identidad étnica, en lo que sigue se presenta un estudio de caso cuyo enfoque es la historia reciente en la aldea de Río Negro, Rabinal, Baja Verapaz. Durante el conflicto armado de 1960 a 1996 en Guatemala, este pueblo sufrió varias masacres, una experiencia que, como voy a demostrar, es importante para la identidad étnica a nivel local.

En los años setenta, el Instituto Nacional de Electrificación de Guatemala (Inde) empezó la construcción de la presa hidroeléctrica Pueblo Viejo-Quixal, Alta Verapaz, la que creó un embalse en el valle del río Chixoy afectando unas 28 aldeas pobladas. Los auspiciadores principales del proyecto eran el Banco Interamericano de Desarrollo (BID) y el Banco Mundial. Una de las comunidades afectadas era la aldea Río Negro o, en achi, la lengua de sus habitantes, Panima'. $\mathrm{Al}$ enterarse de los proyectos del Estado, una parte de los habitantes empezó a oponerse a los planes de traslado de la población por parte del gobierno. Cuando se agudizó la situación, la cual provocó incidentes violentos entre el Inde y la población de la aldea, esta última fue acusada de estar involucrada en actividades guerrilleras; por ello se dirigieron acciones militares contra el asentamiento. En el año 1982, la población casi se extinguió gracias a cuatro masacres en las aldeas de Хосоc, Río Negro, Los Encuentros y Agua Fría. De estas matanzas, en la actualidad se consideran las más importantes las primeras dos. En Xococ, el 13 de febrero, 55 hombres y 19 mujeres fueron asesinados después de haber llegado a este lugar para recuperar sus cédulas de identidad de manos de las autoridades. En Río Negro, el 13 de marzo, 70 mujeres y 107 niños fueron asesinados después de haber sido violados y torturados; 18 niños más fueron secuestrados por miembros de las Patrullas de Autodefensa Civil (PAC) de Xococ para servirles de esclavos en sus residencias. Las siguientes masacres en Los Encuentros y Agua Fría afectaron no sólo a los sobrevivientes de Río Negro refugiados en estos lugares, sino también a individuos procedentes de estas comunidades y de otros asentamientos de la región. Desde entonces la aldea de

\footnotetext{
${ }^{1}$ Los hechos históricos referidos en esta sección son un resumen de la información proveniente de entrevistas con testigos contemporáneos, de los reportes del Equipo de Antropología Forense de Guatemala (1997) y de la Comisión para el Esclarecimiento Histórico (CEH, 1999).
} 
Río Negro quedó abandonada, los sobrevivientes buscaron refugio en la montaña o en lugares lejanos del municipio de Rabinal o se mudaron a la colonia Pacux, Rabinal, construida por el Inde según el plan de reubicación de la población afectada por la represa. Cuando la hidroeléctrica se puso en marcha, grandes partes de los terrenos agrícolas y áreas habitacionales, así como rutas comerciales y sitios arqueológicos, quedaron bajo el agua. Fue hasta principios de los años noventa que varias familias decidieron regresar a su aldea de origen, sobre todo debido a la presión económica y social en el entorno de la cabecera municipal. Construyeron nuevas casas en las partes más altas de Río Negro, arriba del embalse. Actualmente catorce familias viven ahí, se dedican a la agricultura de subsistencia y, en segundo plano, a la venta de pescado y productos agrícolas. Además, algunas familias producen artesanías como metates y textiles.

Por su ubicación en la montaña, el acceso a la aldea es complicado, sobre todo desde los años ochenta, pues la represa inundó las rutas comerciales tradicionales en la orilla del río, así que solamente se puede llegar en lancha; además, el Inde pide un permiso oficial para pasar la represa. El camino a Rabinal se hace a pie y se tarda entre cuatro y ocho horas, dependiendo de la disponibilidad de un transporte motorizado en la aldea de Chitucán a medio camino. La comunicación solamente es posible a través de una radio a la hora concertada. A pesar de la distancia de solamente unos pocos kilómetros a una de las productoras más importantes de energía eléctrica a nivel nacional, la comunidad no tiene acceso a la red de luz eléctrica.

En el año 2008, se inauguró el Centro Histórico y Educativo "Riij Ib’ooy" en Río Negro, un proyecto iniciado por la Agencia Alemana de Cooperación Técnica de carácter educativo y ecoturístico, con el fin de difundir la historia local y de apoyar a la población a través del turismo comunitario autosustentable.

La identidad étnica no se refleja directamente en estos hechos históricos. Varios autores han advertido del peligro de reducir la identidad indígena y maya en Guatemala a la imagen de víctimas de la violencia (Fischer y Benson, 2006: 140; Bastos, 2007: 70, 74-75), pero en este caso no es la historia, o "lo que pasó", lo que determina la identidad, sino la forma como hoy en día se crea la memoria colectiva.

En el caso de Río Negro, la memoria comunicativa de las masacres está en el proceso de tránsito a la memoria cultural. Los incidentes históricos fueron invbestigados por el Equipo de Antropología Forense de Guatemala (1997: véase sobre todo 213-230), la Comisión para el Esclarecimiento Histórico (СЕн, 1999: Tomo VI, 45-64) y el Proyecto Interdiocesano de Recuperación de la Memoria Histórica (Remhi, 1998), entre otros. A partir de los años noventa, se fundaron organizaciones sociales en Rabinal que interceden a favor de los procesos jurídicos contra los responsables, realizan negociaciones de reparación por los daños causados por la hidroeléctrica, así como trabajan para el “...rescate, recuperación de la memoria histórica, cultural, fortalecimiento y promocionando la identidad Maya Achi” (Museo Comunitario Rabinal Achi, 2007), como define su misión el Museo Comunitario de Rabinal. 
Aparte de eso, en 2002 y 2009 se publicaron dos autobiografías de sobrevivientes de Río Negro (Tecú Osorio, 2002; Chen Osorio, 2009). Jesús Tecú Osorio era uno de los niños que estaban presentes durante las masacres y que después fueron llevados a Xococ como esclavos. Carlos Chen Osorio sobrevivió el día de la masacre refugiándose en la montaña donde se escondieron varios años. Ambos autores cuentan su historia desde su infancia hasta la actualidad; ahora son activistas en las organizaciones Fundación Nueva Esperanza Río Negro y Adivima (Asociación para el Desarrollo Integral de las Víctimas de la Violencia en las Verapaces, Maya Achi), respectivamente. Aparte de esto, desde finales de los años noventa varias producciones de documentales audiovisuales han tematizado el conflicto armado en esa región.

Todos estos medios se dirigen a un público heterogéneo. Por una parte, se enfatiza que las publicaciones tendrán un uso histórico y cultural para los locales, que funcionen como "recuerdo de mis padres y memoria para mis hijos", como pone Tecú Osorio en el subtítulo de su autobiografía. El videodocumental Taparon el agua es completamente en achi, lo que confirma este ideal. Las publicaciones de este carácter son el intento consciente de integrar la historia local del conflicto armado en la memoria cultural. Por otra parte, los esfuerzos buscan difundir el (re)conocimiento de los hechos históricos a nivel nacional e internacional. La autobiografía de Tecú Osorio, por ejemplo, ha sido traducida y publicada en inglés y alemán.

En Río Negro el uso de estos medios es casi nulo. Aunque el Centro Histórico y Educativo (CHE) posee una pequeña biblioteca, con materiales literarios y audiovisuales, la historia sigue siendo un objetivo de la tradición oral. La existencia de libros y películas sobre Río Negro es conocida y agradecida, pero medios de difusión aparte del lenguaje no, o tal vez todavía no, juegan un papel importante. Uno de los habitantes mantiene el proyecto personal de redactar una crónica de la aldea que empieza con eventos del año de 1970 y sigue hasta la actualidad. Sin embargo, este proyecto originalmente no se dirigía a la familia o la comunidad, sino principalmente a los visitantes del CHE que vienen de fuera. Para la tradición histórica "interna" el modo oral parece el más eficiente. No obstante, hay otros medios que han logrado importancia para la conciencia histórica: los espacios y los monumentos.

La conexión entre memoria y espacio es fuerte y ha sido objeto de muchas investigaciones. Pierre Nora introdujo el término lieux de mémoire, "sitios de memoria”, para indicar lugares pública y culturalmente asociados a eventos históricos y también espacios mentales, como instituciones, obras artísticas y modelos simbólicos (Nora, 1997: 7). En Río Negro encontramos estos sitios de memoria sobre todo en su forma no metafórica, es decir, lugares espaciales, los que también acentúa Aleida Assmann (1999: 21). Los lugares de la masacre en Río Negro se han convertido en sitios conmemorativos para toda la comunidad, no sólo para la generación que sufrió la violencia, sino también para los jóvenes que han aprendido de los acontecimientos de las masacres. En particular, dos 
puntos en el camino desde la aldea hacia el lugar de la masacre son de alta importancia.

El primero es un nivel en que se encontraban varias casas y un árbol de conacaste. Hoy solamente unas pocas piedras indican las ubicaciones anteriores de estas casas y del conacaste sobreviven los restos quemados del tronco (figura 1). Este lugar es comúnmente referido como el sitio donde los militares y los patrulleros (PAC) obligaron a las mujeres de Río Negro a bailar en un acto de humillación.

El segundo sitio de memoria es Pak'oxom, donde se encontraron los fosos en los que fueron tirados los cuerpos de los masacrados. Varias cruces y tablas con inscripciones recuerdan la fecha de la masacre y los nombres de varias víctimas (figura 2).

Desde que la aldea fue repoblada, todos los 13 de marzo se ha organizado una reunión de conmemoración en ese lugar. En la actualidad, ésta incluye una ceremonia maya nocturna de rezos y ofrendas el 13 y una misa católica el siguiente día. Una marimba toca casi ininterrumpidamente. Estas actividades se deben entender sobre todo en el contexto de la tradición del reconocimiento y de la veneración de los difuntos y los ancestros. Las ceremonias son acompañadas de comida y películas; llegan visitantes de Pacux, de otras comunidades y de organizaciones sociales. Mientras los rituales tienen la función de establecer un espacio propio para los muertos, otras formas de comunicación (tanto las películas como los relatos que se cuentan en esta ocasión entre los testigos y también a los jóvenes y extranjeros) trasmiten los recuerdos de los eventos de la violencia. Así, la conmemoración de la masacre del 13 de marzo se ha convertido en el evento sociocultural más importante del año.

La importancia de estos eventos, no sólo para la generación de víctimas sino también para aquellas que siguen, puede ser explicada a través de su relevancia en la vida cotidiana. La historia de la presa y de las masacres no sólo son cuentos del pasado, sino también, o sobre todo, dan explicaciones para el presente y el futuro. Las condiciones socioeconómicas se juzgan como causas directas de los planes despiadados del Inde y luego de la violencia brutal y arbitraria contra la aldea. El embalse es un recuerdo omnipresente de las tierras fértiles que se quedaron bajo el agua; el área del árbol quemado es testigo de la extensión anterior de la aldea, de su destrucción, su saqueo y de los daños físicos, económicos y psíquicos que nunca han sido reparados. Finalmente, el sitio conmemorativo de Pak'oxom refleja los sufrimientos de las víctimas y sus parientes, pero también el deseo de seguir adelante, de buscar justicia y, para los que viven hoy en Río Negro, de regresar, a pesar de los recuerdos dolorosos, al lugar donde crecieron ellos y sus antepasados.

Entonces, los recuerdos de la violencia no sólo se conservan en discursos orales que principalmente corresponden a la memoria comunicativa, sino también en formas simbólicas que conectan el pasado con tradiciones antiguas, en este caso con el culto de los ancestros que se refleja en las ceremonias de Pak'oxom. 


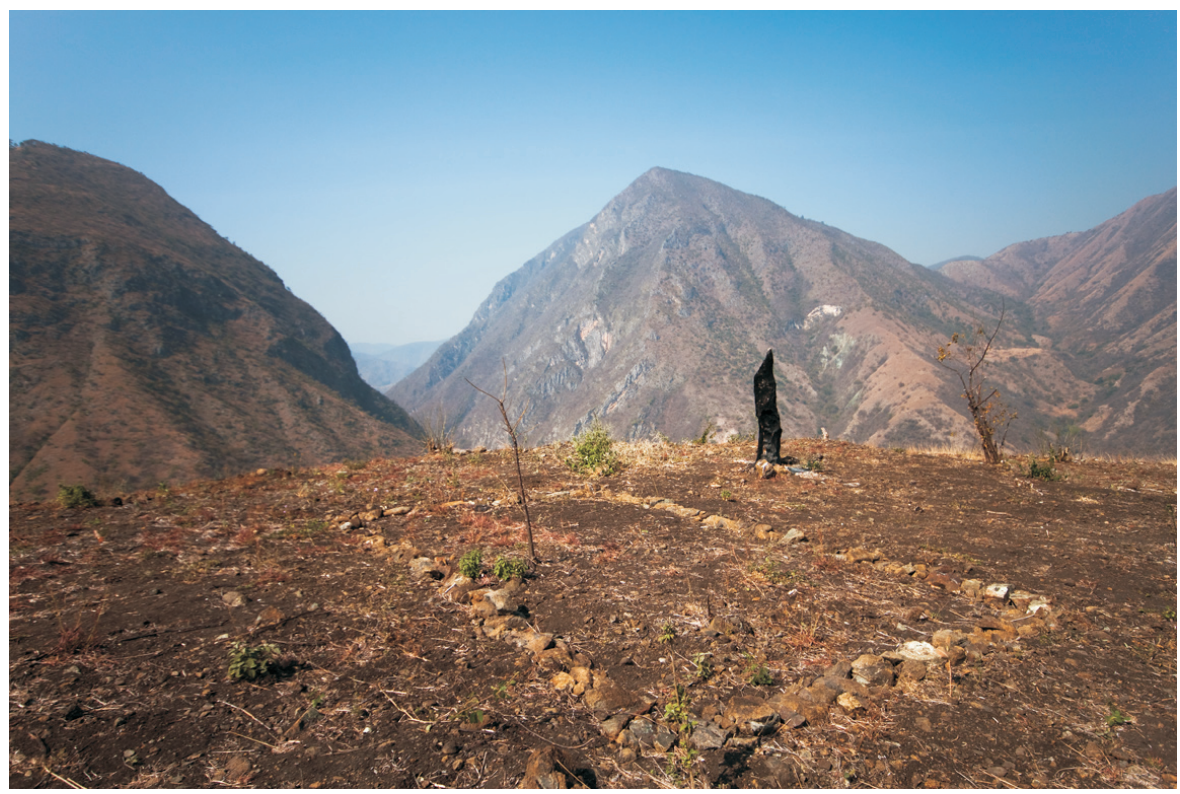

Figura 1. El conacaste quemado. Fotografía del autor

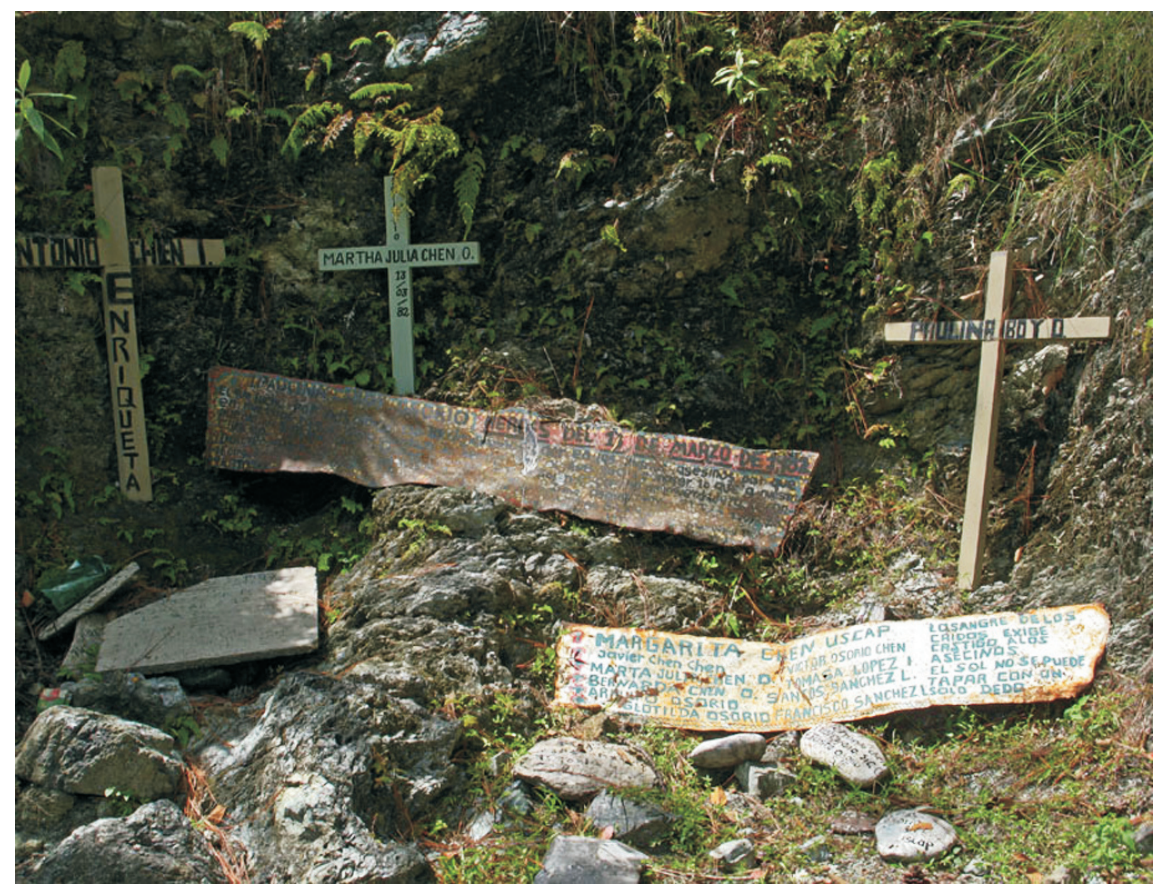

Figura 2. Sitio de memoria en Pak'oxom. Fotografía del autor 
La conmemoración ya forma parte de la cultura local y, por lo tanto, de la memoria cultural, estableciendo una fuerte identificación con la comunidad. ${ }^{2}$

Por ende, se puede advertir que la conmemoración de las masacres de Río Negro es intencional y explícita. Se buscan medios como la tradición oral, la escritura y el ritual para conservar el pasado y prevenir el olvido. Esta actitud pide esfuerzos colectivos para enfrentar el miedo y la injusticia. Sin embargo, todavía hay muchas comunidades en Guatemala que no han logrado una comunicación abierta y pública sobre la época de la violencia. En su estudio sobre las memorias de viudas mayas en El Quiché, Judith Zur describe este proceso en el contexto posconflicto en Guatemala (Zur, 1998: 159-166). Muestra que, en la mitad de los años noventa, el olvido de las experiencias del conflicto armado era lo común entre las viudas cuyos esposos fueron asesinados durante la violencia. A causa de una "política del olvido" por parte del Estado y, sobre todo, por las amenazas e intimidaciones por parte de los militares y patrulleros culpables que todavía estaban muy presentes en la comunidad, muchos preferían el intento de olvidar lo que había pasado. "En consecuencia, el silencio ha sido adaptado como mecanismo de defensa y sobrevivencia en todos los grupos con excepción de unos selectos" (Zur, 1998: 166). Los mismos mecanismos se reflejan en las autobiografías de Jesús Tecú Osorio (2002: 137-138, 141-142, 148) y Carlos Chen Osorio (2009: 145). Cuando empezaron su trabajo de documentación de los casos de violencia en Rabinal, los testigos contaron sus historias titubeantemente, con miedo a los militares. En Río Negro se encuentran varios indicadores para el olvido activo. En Pak'oxom, algunos de los sobrevivientes construyeron una cruz de cemento poco después de la masacre. Esta cruz porta una inscripción que denuncia a los patrulleros de Xococ y al ejército de Guatemala como los culpables de la masacre (figura 3). Poco después, encontraron el monumento quebrado, probablemente a manos de individuos de Xococ que querían eliminar cualquier testimonio de sus acciones. Sin embargo, los sobrevivientes, decididos a no someterse a la represión, reconstruyeron la cruz. Hasta el día de hoy se pueden ver las huellas del intento de destrucción, lo cual indica los intentos de oprimir los testimonios de las víctimas.

Durante las últimas dos décadas, iniciativas de organizaciones no gubernamentales han empezado a estimular el diálogo público sobre la época de guerra. CALDH (Centro para la Acción Legal en Derechos Humanos), Conavigua (Coordinadora Nacional de Viudas de Guatemala), FAFG (Fundación de Antropología Forense de Guatemala) y ECAP (Equipo de Estudios Comunitarios y Acción Psicosocial) son algunas de las instituciones que han contribuido con enfoques diferentes al esclarecimiento histórico, al rompimiento de tabús y al inicio de discursos locales sobre la violencia. No obstante, en muchas partes del país todavía falta mucho trabajo al respecto (cf. Bastos, 2007).

\footnotetext{
${ }^{2}$ Esto no significa que la memoria cultural en Río Negro no va más allá de la violencia. De hecho, se han conservado muchos recuerdos colectivos de tiempos míticos, de la fundación del pueblo y de la vida tradicional.
} 
Río Negro es uno de estos lugares, donde se nota un proceso reflexivo avanzado. Seguramente, un factor importante del esclarecimiento ha sido el trabajo de organizaciones locales como Adivima, pero la existencia de formas rituales de la memoria cultural muestra que al mismo tiempo hay impulsos que salen de la población de las aldeas. En consecuencia, muchos de los habitantes de Río Negro han contado sus experiencias muchas veces y consideran importante trasmitir esta parte de su historia a las próximas generaciones.

\section{Niveles de la identidad étnica}

El ejemplo de Río Negro muestra cómo la integración de elementos de la historia reciente en la memoria cultural funciona en favor de un proceso de "localización" de la identidad étnica. Los elementos de origen más reciente de la cultura en Río Negro, es decir, la conmemoración ritual de las víctimas de las masacres y el establecimiento de sitios de memoria, distinguen a su población, por ejemplo, de la mayoría de los habitantes rabinalenses de la cabecera municipal que, aunque familiarizados con los hechos históricos, no comparten estos rasgos a nivel cultural. Así surge una identidad étnica local basada en rituales y sitios de memoria que obtienen un significado dentro de una comunidad muy restringi-

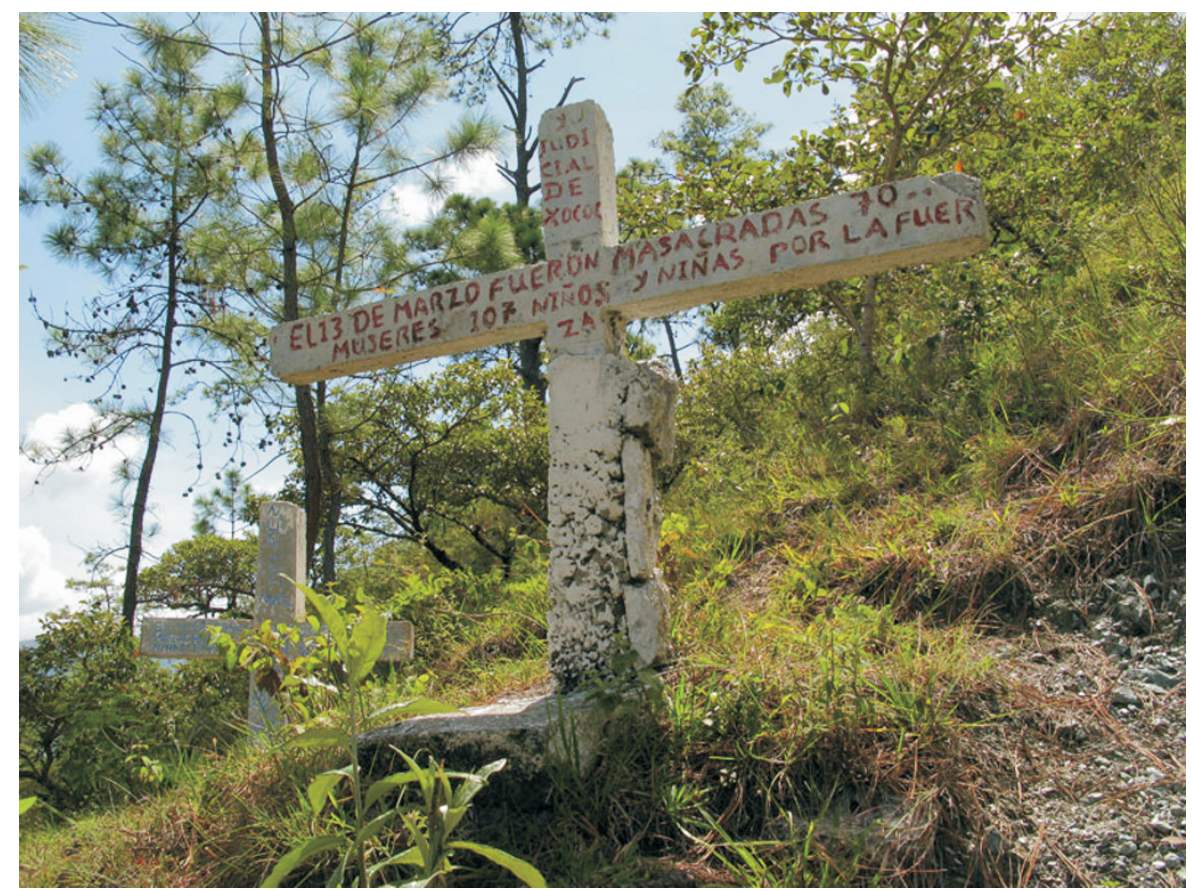

Figura 3. Cruz con inscripción en Pak'oxom. Fotografía del autor 
da. Sin embargo, esta identidad se retroalimenta con los movimientos sociales a nivel municipal y nacional. Un indicador son las publicaciones de testimonios autobiográficos e historiografías que reflejan tanto la afiliación a la cultura local como el deseo de extenderse más allá de las fronteras tradicionales.

Sistematizando los tipos de identidad, podemos constatar que la identidad étnica se expresa en varios niveles distintos de la población. La base de este sistema está formada por las identidades étnicas locales que pueden ser constituidas por pocos individuos como en el caso de la de Río Negro, que hoy incluye básicamente a los sobrevivientes y sus descendientes en Pacux, Rabinal y en la aldea repoblada. La llamo "identidad étnica local" no porque se restrinja a un grupo localmente establecido, sino por los símbolos de origen local en que se basa.

En un nivel más alto se pueden definir varios tipos de identidades étnicas basadas en congruencias, similitudes o intersecciones de identidades locales. Ejemplo de esto es la identidad étnica con base en el estilo de traje o en rasgos linguíísticos. Finalmente, podemos distinguir identidades inter- y supraregionales (como la etnicidad maya), que se definen mediante las congruencias de aquellas identidades locales incluidas en estos niveles. A nivel interregional, la identidad étnica frecuentemente es etnicidad por su definición positivista, y a menudo se forma en contextos sociopolíticos. Sin embargo, debido a la enorme diversidad cultural existente en Guatemala y a las diferencias entre los marcadores étnicos locales, una definición descriptiva para la etnicidad maya sigue siendo un objetivo difícil que, a pesar de la definición oficial a través del Acuerdo, se discute continuamente.

En el proceso de la formación de la etnicidad maya se integran marcadores sincrónicos y asincrónicos como valores tradicionales en la memoria cultural. Los marcadores asincrónicos indican esfuerzos de revitalización mientras los sincrónicos forman la conexión con las identidades étnicas locales.

Por un lado, se puede observar que la etnicidad maya interregional influye a muchas identidades étnicas locales en un proceso de "mayanización". No sólo se refuerzan los marcadores sincrónicos, sino también se introducen asincrónicos (para estudios de caso véase Bastos y Cumes, 2007: Tomo II). Por el otro lado, las identidades étnicas locales siempre han formado una base importante para la etnicidad maya. Los marcadores sincrónicos suelen provenir de aquellas y, además, muchos marcadores asincrónicos sólo poseen un sentido cuando se basan en marcadores sincrónicos establecidos. ${ }^{3}$ Entonces, se puede decir que las identidades mayas locales y la etnicidad maya interregional se distinguen cualitativamente. Sin embargo, interactúan y se retroalimentan (figura 4). Esto

\footnotetext{
${ }^{3}$ Por ejemplo, el uso de silabogramas jeroglíficos requiere una lengua que es, por lo menos en su mayor parte, fonéticamente compatible. Se podrían desarrollar silabogramas para fonemas no existentes en el maya clásico, pero otros obstáculos como grupos consonánticos de tres consonantes en el español formarían problemas estructurales.
} 


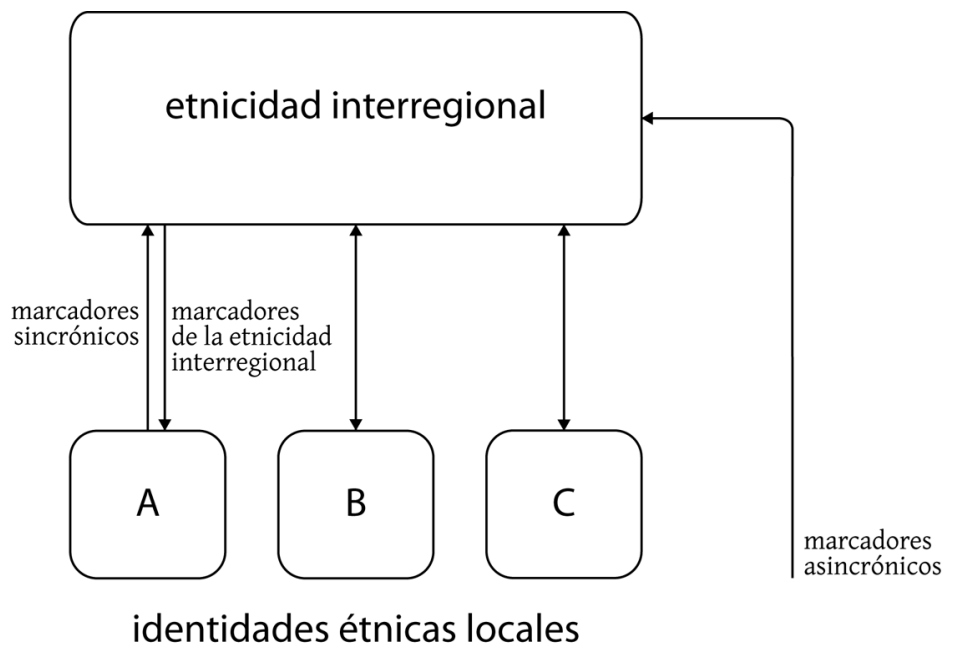

Figura 4. Interacción de las identidades étnicas

se aprecia, por ejemplo, en la manera en que los sistemas semióticos de ambos niveles se relacionan.

A primera vista, la mayanización en Río Negro casi no parece presente. Esto se debe principalmente a su aislamiento geográfico y comunicativo. Pero en muchos detalles ya se pueden detectar rasgos que reflejan marcadores de la etnicidad maya. Talleres de organizaciones sociales en la aldea promueven los valores del movimiento maya y también la presentación pública del Centro Histórico y Educativo incluye ideales mayas como el orgullo étnico y la importancia de las tradiciones.

Los procesos en la cabecera municipal de Rabinal ya muestran claramente la presencia del movimiento maya. Instituciones como el Museo Comunitario Rabinal Achi proveen material educativo y exponen tanto piezas representativas de las culturas materiales locales del pasado y de la actualidad, como imágenes de las víctimas del conflicto armado y explicaciones de la historia reciente. Organizaciones como Adivima se identifican específicamente con los "maya achi”, una forma de expresión que construye la unidad linguiísticorregional y que al mismo tiempo refleja la idea de la solidaridad y permanencia mayas. Además, se refuerza la educación cultural a través de instituciones como la Academia de Lenguas Mayas de Guatemala y proyectos como la escuela de la Fundación Nueva Esperanza, en Río Negro, que mantiene una escuela bilinguie donde se ha incluido en su plan de estudios asuntos culturales y temas de la historia de la violencia.

En el caso de la aldea actual de Río Negro las formas de conmemorar al pasado se caracterizan por su relación local, pero sus estructuras tienen muchos paralelismos en todo el país y ya empiezan a formar un marcador sincrónico de la etnicidad maya. Diferentes comunidades en toda Guatemala desarrollan es- 
trategias culturales para superar las experiencias traumáticas de la violencia, un motivo importante de los movimientos mayas (Bastos, 2007: 74-75). En el caso especial de Río Negro la identidad étnica local se ha cambiado drásticamente desde los años ochenta. Las estrategias para seguir viviendo con las experiencias de miedo, violencia y desamparo incluyen la creación de espacios de conmemoración y la publicación y difusión de su historia. En este sentido, se puede ver a Río Negro como un ejemplo positivo para comunidades que quieren superar el terror a través de la reflexión sobre la historia.

\section{Conclusiones}

La etnicidad como forma consciente y funcional de la identidad étnica se expresa a través de marcadores sincrónicos y asincrónicos. Estos marcadores forman el sistema semiótico que constituye la cultura que define la etnicidad. Tratándose de etnicidades interregionales, como en el caso de la etnicidad maya en Guatemala, los marcadores sincrónicos tienden a provenir de identidades étnicas locales, mientras los marcadores asincrónicos son retomados de discursos no locales. Por esta conexión las identidades interregionales y locales se retroalimentan, lo cual resulta en el reforzamiento de elementos tradicionales y la introducción de elementos nuevos o revitalizados a nivel local.

El Acuerdo sobre identidad y derechos de los pueblos indígenas normaliza la etnicidad maya a través de una lista de marcadores que provienen tanto de debates académicos como de las culturas locales actuales. Se puede entender como reflejo de la construcción de la etnicidad del movimiento maya, aunque no necesariamente representa las ideas de todos sus actores en la actualidad. Los aspectos primordiales y constructivistas forman partes débiles de la "definición" de la identidad; sin embargo, el Acuerdo sigue siendo la base de argumentos políticos en cuanto a la legitimación del sentimiento colectivo de ser maya.

El proceso de la mayanización consiste, básicamente, en la extensión de los marcadores normativos a las identidades étnicas locales. No obstante, siempre hay que considerar que la influencia no es unilateral sino mutual. Los marcadores de las identidades étnicas locales también se involucran en los discursos interregionales de la etnicidad. Este proceso se puede ilustrar a través de la formación de una memoria cultural de la violencia de los años ochenta. No se refiere a la victimización de los grupos mayas, sino a las estrategias tradicionales de conmemorar a los difuntos que fueron asesinados en el contexto de la guerra civil. El ejemplo de Río Negro demuestra cómo dentro de la aldea se trasmite esta memoria por medio de la tradición oral, rituales y sitios de memoria, aunque al mismo tiempo hay esfuerzos para distribuir estos recuerdos a nivel nacional e internacional. Procesos similares se encuentran en otras comunidades en Guatemala, frecuentemente a causa del apoyo de organizaciones institucionalizadas. Así, las estructuras de la conmemoración y la memoria colectiva se están incorporando 
al canon de marcadores étnicos no sólo de las culturas locales, sino también de la etnicidad maya interregional. Para entender mejor los procesos actuales de la construcción de identidades en contextos pluriculturales y globalizadores, nuestro enfoque en el futuro debe centrarse más en el análisis de esta interacción de estructuras culturales locales con los valores étnicos a nivel interregional.

\section{BIBLIOGRAFÍA}

Alejos García, José

2008 "Etnicidad maya en un mundo global", El territorio maya. Memoria de la Quinta Mesa Redonda de Palenque, Rodrigo Liendo Stuardo (ed.). México: Instituto Nacional de Antropología e Historia, 191-210.

Assmann, Aleida

1999 Erinnerungsräume: Formen und Wandlungen des kulturellen Gedächtnisses. Munich: C.H. Beck (C.H. Beck Kulturwissenschaft).

2008 "Canon and Archive", Cultural Memory Studies: An International and Interdisciplinary Handbook, Astrid Erll, Ansgar Nünning y Sara B. Young (eds.). Berlín/ Nueva York: Walter de Gruyter, 97-107.

Assmann, Jan

1992 Das kulturelle Gedächtnis: Schrift, Erinnerung und politische Identität in frühen Hochkulturen. Munich: C.H. Beck (C.H. Beck Kulturwissenschaft).

Barth, Fredrik

1969 "Introduction”, Ethnic Groups and Boundaries: The Social Organization of Culture Difference, Fredrik Barth (ed.). Bergen / Oslo / Tromsö [Noruega]: Universitetsforlaget, 9-38.

Bastos, Santiago

2007 "Violencia, memoria e identidad: el caso de Choatalum (San Martín Jilotepeque, Chimaltenango)", Mayanización y vida cotidiana. La ideología multicultural en la sociedad guatemalteca, Santiago Bastos y Aura Cumes (eds.). Guatemala: Facultad Latinoamericana de Ciencias Sociales / Centro de Investigaciones Regionales de Mesoamérica / Fundación Cholsamaj, 45-78.

Bastos, Santiago y Manuela Camus

2003 Entre el mecapal y el cielo. Desarrollo del movimiento maya en Guatemala. Guatemala: Facultad Latinoamericana de Ciencias Sociales / Fundación Cholsamaj.

Bastos, Santiago y Aura Cumes (eds.)

2007 Mayanización y vida cotidiana. La ideología multicultural en la sociedad guatemalteca. Guatemala: Facultad Latinoamericana de Ciencias Sociales / Centro de Investigaciones Regionales de Mesoamérica / Fundación Cholsamaj, 3 vols. 
CEH (Comisión para el Esclarecimiento Histórico)

1999 Guatemala: Memoria del silencio. Guatemala: F \& G Editores, 12 vols.

Chen Osorio, Carlos

2009 Historias de lucha y de esperanza. Rabinal: Asociación para el Desarrollo Integral de las Víctimas de la Violencia en las Verapaces, Maya Achi.

Equipo de Antropología Forense de Guatemala

1997 Las masacres en Rabinal. Estudio histórico antropológico de las masacres de Plan de Sánchez, Chichupac y Río Negro, 2a. ${ }^{a}$ ed. Guatemala: Equipo de Antropología Forense de Guatemala.

Fischer, Edward F.

2001 Cultural Logics and Global Economics: Maya Identity in Thought and Practice. Austin: University of Texas Press.

Fischer, Edward F. y Peter Benson

2006 Broccoli and Desire. Global Connections and Maya Struggles in Postwar Guatemala. Stanford: Stanford University Press.

Fischer, Edward F. y R. McKenna Brown (eds.)

1996 Maya Cultural Activism in Guatemala. Austin: University of Texas Press.

Fischer, Edward F. et al.

1999 "Cultural Logic and Maya Identity: Rethinking Constructivism and Essentialism [and Comments and Reply]", Current Anthropology, 40 (4): 473-499. Chicago: University of Chicago Press.

Geertz, Clifford

1973 The Interpretation of Cultures: Selected Essays. Nueva York: Basic Books.

Halbwachs, Maurice

1992 On collective memory. Chicago: University of Chicago Press (The Heritage of Sociology).

Macleod, Morna

2006 "De brechas a puentes: Las reivindicaciones de identidad y cultura en el movimiento maya en Guatemala”, Dialogando alteridades. Identidades y poder en Guatemala, José Alejos García (ed.). México: Universidad Nacional Autónoma de México, Instituto de Investigaciones Filológicas, Centro de Estudios Mayas, 39-82.

Museo Comunitario Rabinal Achi

2007 “Objetivos”, Museo Comunitario Rabinal Achi <http://www.museo.rabinal. info/objetivos.html > [Consultada el 17 de septiembre del 2010.]

Naciones Unidas, Gobierno de Guatemala y Unidad Revolucionaria Nacional Guatemalteca 1995 Acuerdo sobre identidad y derechos de los pueblos indígenas: punto 3 del Acuerdo 
de paz firme y duradera: suscrito en Ciudad de México por el Gobierno de la República de Guatemala y la Unidad Revolucionaria Nacional Guatemalteca, 31 de marzo de 1995. Guatemala: Cholsamaj.

Nelson, Diane M.

1996 "Maya Hackers and the Cyberspatialized Nation-State: Modernity, Ethnostalgia, and a Lizard Queen in Guatemala", Cultural Anthropology, 11 (3): 287308. Arlington: American Anthropological Association

Nora, Pierre

1997 Zwischen Geschichte und Gedächtnis. Berlín: Klaus Wagenbach.

Olick, Jeffrey K.

1999 "Collective Memory: The Two Cultures", Sociological Theory, 17 (3): 333-348. Washington, D.C.: American Sociological Association.

Otzoy, Irma

1996 "Maya Clothing and Identity", Maya Cultural Activism in Guatemala, Edward F. Fischer y R. McKenna Brown (eds.). Austin: University of Texas Press, 141-155.

Remhi (Proyecto Interdiocesano de Recuperación de la Memoria Histórica)

1998 Guatemala: nunca más. Guatemala: Oficina de Derechos Humanos del Arzobispado de Guatemala, 4 vols.

Sachse, Frauke (ed.)

2006 Maya Ethnicity: The Construction of Ethnic Identity from Preclassic to Modern Times. Bonn: Antoin Saurwein (Acta Mesoamericana, 19).

Schele, Linda y Nikolai Grube

1996 "The Workshop for Maya on Hieroglyphic Writing", Maya Cultural Activism in Guatemala, Edward F. Fischer y R. McKenna Brown (eds.). Austin: University of Texas Press, 131-140.

Sturm, Circe

1996 "Old Writing and New Messages: The Role of Hieroglyphic Literacy in Maya Cultural Activism", Maya Cultural Activism in Guatemala, Edward F. Fischer y R. McKenna Brown (eds.). Austin: University of Texas Press, 114-130.

Tecú Osorio, Jesús

2002 Memoria de las masacres de Río Negro. Recuerdo de mis padres y memoria para mis hijos. Rabinal: Fundación Nueva Esperanza, Río Negro, Rabinal.

Vansina, Jan

1985 Oral Tradition as History. Madison: The University of Wisconsin Press.

Warren, Kay B.

1998 Indigenous Movements and their Critics: Pan-Maya Activism in Guatemala. Princeton: Princeton University Press. 
Weber, Max

1964 Economía y sociedad, $2^{\mathrm{a}}$ ed. en español. México: Fondo de Cultura Económica (Esbozo de Sociología Comprensiva, 3).

Welzer, Harald

2002 Das kommunikative Gedächtnis: Eine Theorie der Erinnerung. Munich: C.H. Beck.

Yinger, J. Milton

1985 "Ethnicity", Annual Review of Sociology, 11: 151-180. Danvers [Estados Unidos]: Clearance Center.

Zur, Judith N.

1998 Violent Memories: Mayan War Widows in Guatemala. Boulder [Estados Unidos]: Westview Press. 